\title{
Polarization-Converting Nanoantennas for Light Absorption Enhancement in Anisotropic 2D Black Phosphorus
}

\author{
Nima Sefidmooye Azar ${ }^{1}$, James Bullock ${ }^{1}$, Sivacarendran Balendhran ${ }^{2}$, Hyungjin Kim ${ }^{3,4}$, Ali Javey ${ }^{3,4}$, and \\ Kenneth B. Crozier ${ }^{1,2,5^{*}}$ \\ 1 Department of Electrical and Electronic Engineering, University of Melbourne, Victoria 3010, Australia \\ 2 School of Physics, University of Melbourne, Victoria 3010, Australia \\ 3 Department of Electrical Engineering and Computer Sciences, University of California, Berkeley, CA 94720, USA \\ 4 Materials Sciences Division, Lawrence Berkeley National Laboratory, Berkeley, CA 94720, USA \\ 5 ARC Centre of Excellence for Transformative Meta-Optical Systems, University of Melbourne, Victoria 3010, Australia \\ *e-mail address:kcrozier@unimelb.edu.au
}

\begin{abstract}
Optical anisotropy and thinness of 2D black phosphorous (bP) lead to weak light absorption, which limits the performance of bP-based photodetectors. We demonstrate that Tshaped nanoantennas can be integrated with bP to overcome this issue. (C) 2020 The Author(s)
\end{abstract}

\section{Introduction}

Multilayer black phosphorous (bP), a two-dimensional (2D) layered material, has shown great potential for infrared (IR) photodetection owing to its direct and narrow bandgap of $\sim 0.31 \mathrm{eV}[1,2]$. However, a major challenge for photodetectors based on $\mathrm{bP}$ and other 2D materials is their low light absorption which results from their thinness. In addition, bP has an anisotropic crystal structure with an armchair atomic arrangement along one direction and a zigzag arrangement along the other. The optical absorption in the armchair direction is much stronger than that in the zigzag direction [3]. Although this property can be exploited for some applications [3], in general, it is not desirable. For example, photodetectors based on bP are nearly blind to one polarization. This also means that the power carried by one polarization cannot be collected when detecting unpolarized light. Herein, we demonstrate Tshaped plasmonic nanoantennas atop optical cavity substrates for enhancing the light absorption in multilayer bP in the IR region. T-shaped nanoantennas convert the light polarization from the zigzag to armchair crystal direction. They also confine light in the antenna gap which leads to strong near-field intensity and thus light absorption enhancements. T-shaped nanoantennas were previously proposed as a bimodal antenna structure with two independent resonances localized in a single nanogap [4].

\section{Method}

The finite-difference time-domain (FDTD) technique, implemented in the Lumerical FDTD Solutions software package, was employed to solve the Maxwell's equations. The light source was a broadband $(\lambda=1.5-4.5 \mu \mathrm{m})$ linearly polarized plane wave at normal incidence. The refractive index values of bP were taken from ref. [3].

\section{Results and Discussions}

The crystal structure of bP is visualized in Fig. 1 (a). The armchair and zigzag crystal directions are defined as $x$ and $y$, respectively. Fig. 1 (b) plots the optical power absorbed in a 10-nm-thick bP layer sitting on an $\mathrm{SiO}_{2} / \mathrm{Si}$ substrate $\left(\mathrm{SiO}_{2}\right.$ thickness $\left.=300 \mathrm{~nm}\right)$ for $x$ - and $y$-polarized light. This configuration is referred to as " $\mathrm{SiO}_{2} / \mathrm{Si}$ " here. As expected, the absorption for $x$-polarized light is much greater than that for $y$-polarized light. Fig. 1 (c) illustrates the "T-Shaped" configuration, which is formed by integrating periodic T-shaped Au plasmonic nanoantennas with a 10nm-thick bP sitting on an $\mathrm{Al}_{2} \mathrm{O}_{3} / \mathrm{Au}$ optical cavity substrate. Geometrical parameters of T-shaped nanoantennas are depicted in Fig. 3 (d). The antenna gap is aligned in the $x$ (armchair) direction. Fig. 3 (e) presents the optical power absorbed in bP with varying $l_{x}$ for $x$ - and $y$-polarized incident light. Antenna thickness and gap $(g)$ were set to $30 \mathrm{~nm}$ and $40 \mathrm{~nm}$, respectively. Other parameters $\left(l_{y}, t_{s}\right.$, and $\left.w\right)$ were optimized for unpolarized light absorption. E.g. for $l_{x}$ $=400 \mathrm{~nm}$, we have $\left(l_{y}, t_{s}, w\right)=(290,120,120) \mathrm{nm}$. High absorption values are achieved for both polarizations. For instance, for $l_{x}=400 \mathrm{~nm}$, the bP absorption takes value of $66.76 \%$ and $49.94 \%$ for $x$ - and $y$-polarized illumination, respectively, at $\lambda=3.08 \mu \mathrm{m}$. These are enhancements of 9.4 and 185 times for $x$ - and $y$-polarized illumination, respectively, in comparison with the commonly used " $\mathrm{SiO}_{2} / \mathrm{Si}$ " structure.

Fig. 2 (a) presents the light absorption density in bP in the "T-Shaped; $l_{x}=400 \mathrm{~nm}$ " configuration for $x$ polarized illumination at $\lambda=3.08 \mu \mathrm{m}$. Results for $y$-polarized illumination are shown in Fig. 2 (b). For $x$-polarized illumination, T-shaped nanoantennas act like regular rod nanoantennas; they enhance the light intensity and thus 
absorption in the antenna gap through localized surface plasmon resonances. For $y$-polarized illumination, in addition to intensity enhancement, the T-shaped nanoantennas rotate the polarization to the $x$ direction (along which bP can absorb light) in the gap and around the T legs.
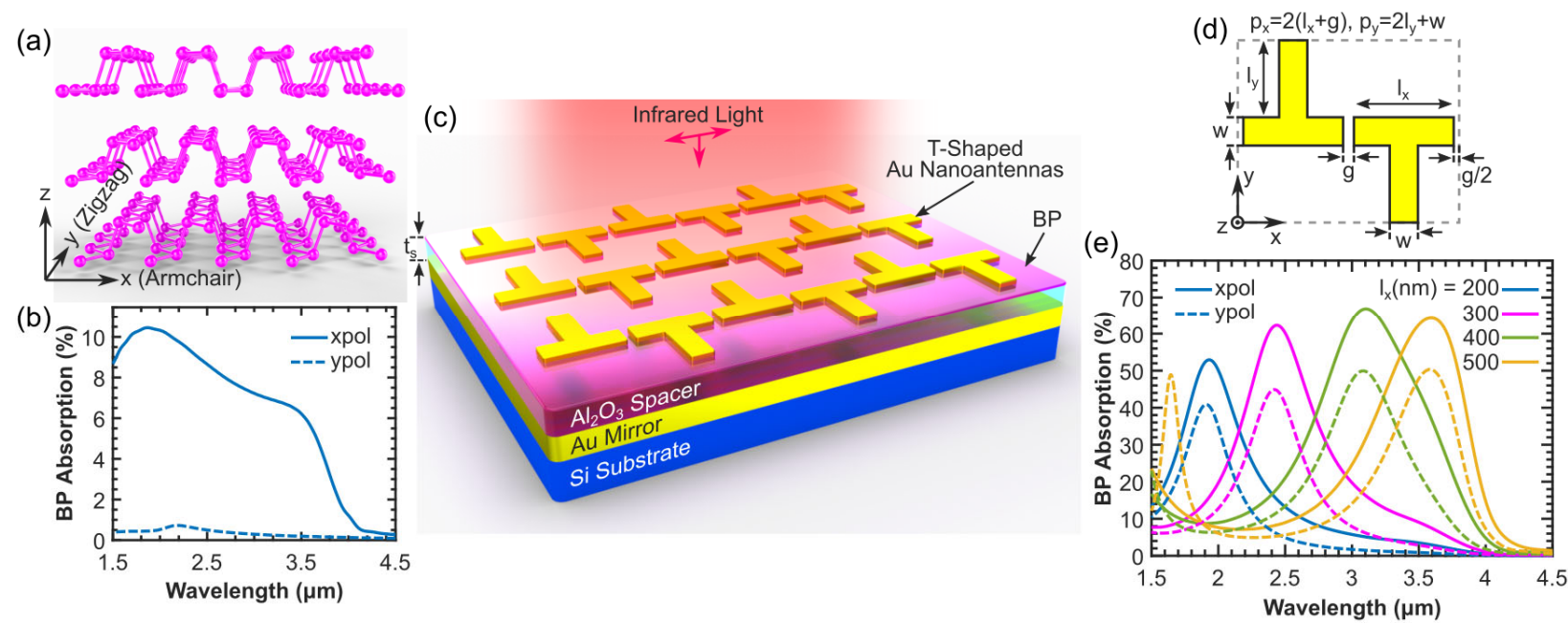

Fig. 1. (a) Schematic of bP crystal structure. (b) Optical power absorbed in bP in the " $\mathrm{SiO}_{2} / \mathrm{Si}$ " structure for $x$ - and $y$-polarized light. (c) Schematic illustrating "T-Shaped" structure. (d) Geometrical parameters of T-shaped nanoantennas. The grey dashed box represents the unit cell. $p_{x}$ and $p_{y}$ are the period in the $x$ and $y$ directions, respectively. (e) Optical power absorbed in bP in the "T-Shaped" structure with varying $l_{x}$ for $x$ and $y$-polarized illumination.

(a) BP Absorption Density $\left(\% . \mathrm{nm}^{-3}\right)$

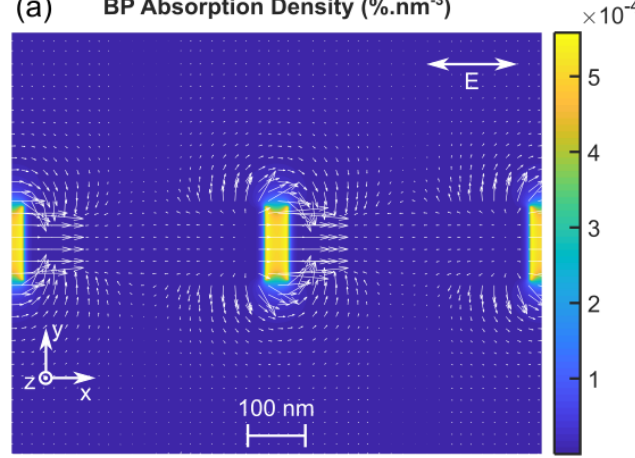

(b) BP Absorption Density $\left(\% . \mathrm{nm}^{-3}\right)$

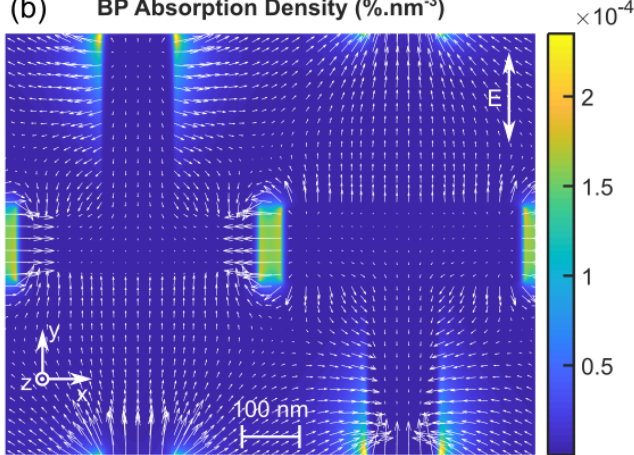

Fig. 2. Light absorption density in bP in the "T-Shaped" structure with $l_{x}=400 \mathrm{~nm}$ for (a) $x$-polarized and (b) $y$-polarized incident light at $\lambda=$ $3.08 \mu \mathrm{m}$. The arrows represent the in-plane component of electric field at an instant of time. The absorption density and electric field values depicted here are the average values across the thickness of bP.

\section{Conclusion}

In this work, we demonstrated that T-shaped nanoantenna-cavity hybrid can efficiently enhance the IR light absorption in multilayer bP by boosting the light intensity and converting its polarization. BP has great potential for IR detection, and its integration with the optical structure presented here can significantly boost the performance of devices based on this material.

\section{References}

[1] H. Liu, Y. Du, Y. Deng, and D. Y. Peide. "Semiconducting Black Phosphorus: Synthesis, Transport Properties and Electronic Applications." Chem. Soc. Rev. 44, 2732-2743 (2015).

[2] X. Chen, X. Lu, B. Deng, O. Sinai, Y. Shao, C. Li, S. Yuan et al. "Widely Tunable Black Phosphorus Mid-Infrared Photodetector." Nat. Commun. 8, 1-7 (2017).

[3] J. Bullock, M. Amani, J. Cho, Y.-Z. Chen, G. H. Ahn, V. Adinolfi, V. R. Shrestha et al. "Polarization-Resolved Black Phosphorus/Molybdenum Disulfide Mid-Wave Infrared Photodiodes with High Detectivity at Room Temperature.” Nat. Photonics 12, 601-607 (2018).

[4] K. Dopf, C. Moosmann, S. W. Kettlitz, P. M. Schwab, K. Ilin, M. Siegel, U. Lemmer, and H.-J. Eisler. "Coupled T-Shaped Optical Antennas with Two Resonances Localized in a Common Nanogap." ACS Photonics 2, 1644-1651 (2015). 


\section{University Library}

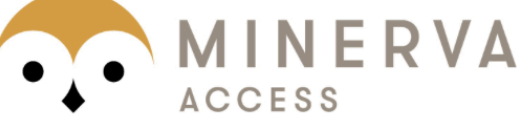

A gateway to Melbourne's research publications

Minerva Access is the Institutional Repository of The University of Melbourne

Author/s:

Azar, NS;Bullock, J;Balendhran, S;Kim, H;Javey, A;Crozier, KB

Title:

Polarization-Converting Plasmonic Nanoantennas for Light Absorption Enhancement in Anisotropic 2D Black Phosphorus

Date:

2021-01-01

\section{Citation:}

Azar, N. S., Bullock, J., Balendhran, S., Kim, H., Javey, A. \& Crozier, K. B. (2021).

Polarization-Converting Plasmonic Nanoantennas for Light Absorption Enhancement in Anisotropic 2D Black Phosphorus. 2021 CONFERENCE ON LASERS AND ELECTROOPTICS (CLEO), IEEE. https://doi.org/10.1364/cleo_qels.2021.fth2k.7.

Persistent Link:

http://hdl.handle.net/11343/294861 